\section{CERCOS PERIMETRALES EN PIEZAS COMPRIMIDAS DE SECCION CIRCULAR}

\author{
José Ramón Rosso Ródenas* \\ Ingeniero Técnico de Obras Públicas.
}

$452-8$

\section{RESUMEN}

Se da una regla práctica que, de acuerdo con el dimensionamiento de cercos de la EH 80 , permite el cálculo inmediato de cercos perimetrales en piezas comprimidas de hormigón armado.

La Instrucción para el proyecto y la ejecución de obras de hormigón en masa o armado, EH-80, mantiene para el dimensionamiento y disposición de cercos en piezas o zonas de secciones sometidas a compresión los mismos criterios que la EH-73. Es decir, la separación máxima entre cercos será de 15 veces el menor diámetro de barra longitudinal comprimida y el diá. metro del cerco será, como mínimo, la cuarta parte del mayor diámetro.

Esta regla, complementada con otras referentes a la armadura principal, es de fácil aplicación en secciones rectangulares u otras de lados planos. Pero cuando la sección de la pieza es circular la armadura de cercos se complica enormemente si queremos evitar, con igual criterio, el pandeo de todas las barras. Una disposíción posible sería la de la figura 1, en la que puede obsenarse la gran cuantía de armadura transversal que resultaría, aun sin contar con un cerco exterior circular completo que, por motivos constructivos, sería conveniente disponer.

Parece que los cercos racionalmente más apropiados, desde los puntos de vista de ejecución y económico, serían los circulares bien a base de unidades independientes bien en hélice. Pero ¿será posible impedir el pandeo de la armadura principal empleando única. mente cercos circulares perimetrales? La demostración teórica de que sí es posible, basándonos estricta.

\footnotetext{
* Empresa: Ibérica de Ingeniería y Organización (IBERING)
}

mente en el articulado de la EH.80, es el objeto de este artículo. La demostración práctica - sin pretender con ello, ni mucho menos, que ésta sea una prueba experimental en toda regla - la podemos encontrar en los fustes de numerosos puentes y viaductos proyectados por IBERING, S. A . con este criterio y que, una vez en servicio, no han presentado absolutamente ningún problema. La misma regla de dimensionado de cercos circulares se ha hecho extensiva a los pilotes pero, naturalmente, su buen resultado en ellos no queda tan visible como en los fustes.

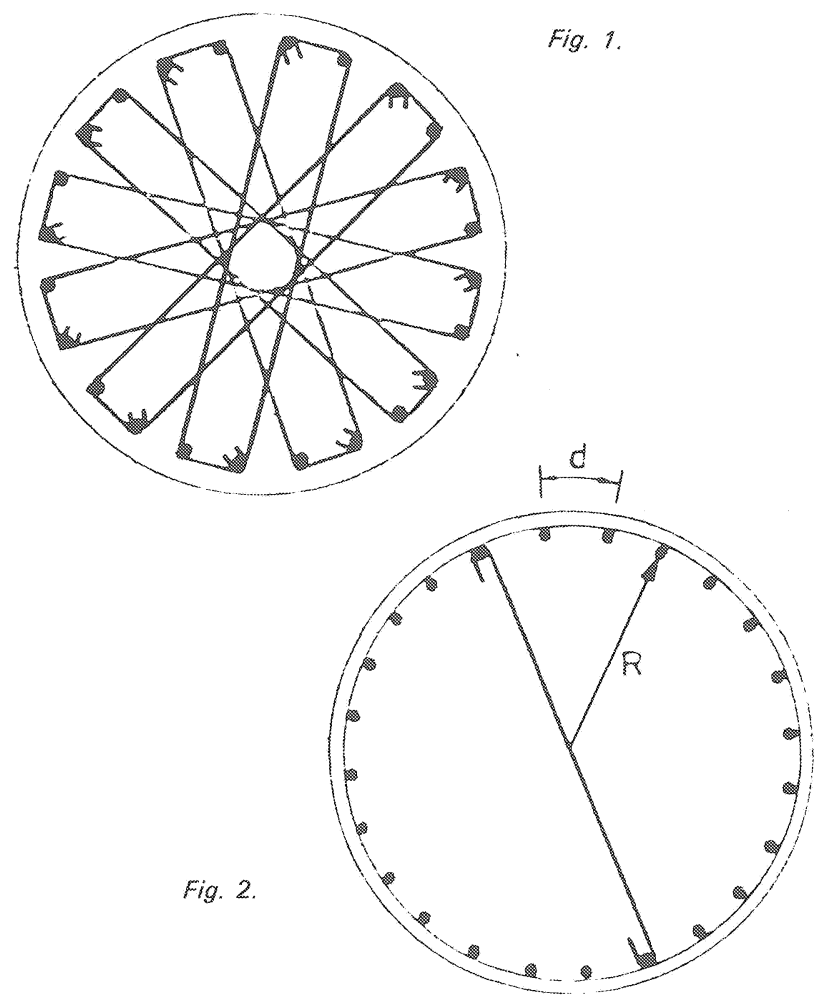

Supongamos una sección como la de la figura 2 , en la que se han dispuesto $n$ barras de diámetro $\varnothing$. El error cometido será despreciable y tendremos, en cambio, la ventaja de una gran simplificación si consideramos que el cerco en lugar de ser exterior a las barras principales pasa por los ejes de éstos. Sea $R$ el radio del cerco así considerado.

Es evidente que un tirante como el indicado en la figura 2 de diámetro $\varnothing \div 4$ y situado cada $15 \varnothing$ impedirá el pandeo, según la EH-80, de las barras que sujeta. La fuerza que este tirante podrá llegar a desarrollar será:

$$
P=\frac{\varnothing^{2}}{64} \pi f_{y d}
$$


siendo $f_{\text {y }}$ la resistencia de cálculo del acero.

Si $d$ es la distancia según el arco entre dos barras consecutivas y suponemos que todas las barras tienen igual disposición de tirantes se desarrollará una fuerza por metro de cerco de:

$$
\begin{gathered}
p=\frac{p}{d}=\frac{\varnothing^{2}}{64} \pi f_{y d} \cdot \frac{1}{d} \\
y \text { como } d=\frac{2 \pi R}{n} p=n \frac{\varnothing^{2}}{128 R} f_{y d}
\end{gathered}
$$

Si suprimimos los tirantes esta fuerza de desviación tendría que ser soportada por el arco circular $y$, por consiguiente, se desarrollaría en él una tracción que sería:

$$
T=n \frac{\varnothing^{2}}{128 R} f_{y d} \cdot R=\frac{n \varnothing^{2}}{128} f_{y d}
$$

La sección del cerco tendría que ser de $\frac{T}{f_{y d}}=$ $=\frac{n \varnothing^{2}}{128}$ y su diámetro $\varnothing_{\mathrm{t}}$ será:

$\varnothing_{t}=\sqrt{\frac{n \varnothing^{2}}{128 \cdot \frac{\pi}{4}}}=\frac{\varnothing}{4} \sqrt{\frac{n}{2 \pi}}$

Ahora bien, si llamamos $A_{\text {tot a la sección total de }}$ barras longitudinales del pilar tendremos:

$$
A_{\text {tot }}=n \pi \frac{\varnothing^{2}}{4}
$$

y la sección de un cerco referida a esta $A_{\text {tot }}$ será:

$$
\begin{aligned}
& \frac{\frac{\varnothing^{2}}{16} \cdot \frac{n}{2 \pi} \pi \cdot \frac{1}{4}}{n \pi \frac{\varnothing^{2}}{4}}= \\
& =\frac{1}{32 \pi}=0,00995 \sim 0,01
\end{aligned}
$$

Es decir, que como regla práctica se puede enunciar:

La separación máxima entre cercos perimetrales de secciones circulares será de 15 veces el diámetro de las barras en compresión, supuestas todas ellas con igual diámetro, debiendo ser la sección del cerco igual - mayor que el uno por ciento del total de la armadura principal. Si la separación 'S' entre cercos es inferior a 15 diámetros su diámetro $\varnothing$, podrá disminuirse de tal forma que la relación entre la sección del cerco y la separación 'S' siga siendo la misma.
Por consiguiente, en general, la sección de un cerco con separaciones ' $S$ ' será:

$$
A_{c}^{s}=\frac{0,01}{15 \varnothing} A_{\text {tot }} \cdot S
$$

Realizaremos ahora un estudio comparativo entre las cantidades de armadura transversal empleadas según el sistema de la figura 1 y el de la figura 2.

Longitud de un cerco sin contar patillas:

$$
\begin{gathered}
L=2(2 c+d) \quad d \sim \frac{2 \pi R}{n} \\
L=2\left[2 \sqrt{R^{2}-\frac{\pi^{2} R^{2}}{n^{2}}}+\frac{2 \pi R}{n}=1\right. \\
=4 R\left[\sqrt{\left.1-\frac{\pi^{2}}{n^{2}}+\frac{\pi}{n}\right]}\right.
\end{gathered}
$$

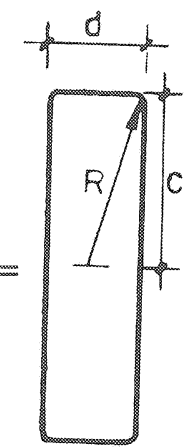

Longitud de $\frac{n}{4}$ cercos:

$L_{t}=n R\left[\sqrt{1-\frac{\pi^{2}}{n^{2}}}+\frac{\pi}{n}\right]$

Con el diámetro teórico de $\frac{\varnothing}{4}$ el volumen total del hierro de un plano de cercos será:

$V_{1}=\frac{\varnothing^{2}}{16} \cdot \frac{\pi}{4} \cdot n R\left[\sqrt{1-\frac{\pi^{2}}{n^{2}}}+\frac{\pi}{n}\right]$

El volumen de acero de un cerco circular, con sección del $1 \%$ de la armadura principal será:

$$
\begin{aligned}
& v_{2}=0,01 \cdot n \frac{\varnothing^{2}}{4} \cdot \pi \cdot 2 \pi R \\
& \frac{V_{1}}{V_{2}}=\frac{\sqrt{1-\frac{\pi^{2}}{n^{2}}+\frac{\pi}{n}}}{0,16 \times 2}
\end{aligned}
$$

La siguiente tabla nos da la relación de $\frac{V_{1}}{V_{2}}$ para diferentes valores de $n$

$\begin{array}{cccccccc}n & 8 & 12 & 16 & 20 & 24 & 100 & \infty \\ V_{1} N_{2} & 1,31 & 1,22 & 1,17 & 1,14 & 1,12 & 1,03 & 1\end{array}$


Vemos que siempre la armadura de la primera disposición es superior a la de la segunda, y esto sin contar con una armadura circular de piel que sería conveniente colocar en el primer caso.

En conclusión las ventajas fundamentales del cerco perimetral, como armadura transversal única, son esencialmente las siguientes:

\section{- Simplicidad constructiva}

- Una mejor calidad de la obra ejecutada, pues una disposición de estribado distinta produciría segregación del hormigón.

- Menor cuantía de acero.

\section{última publicación del I.E.T.c.c.}

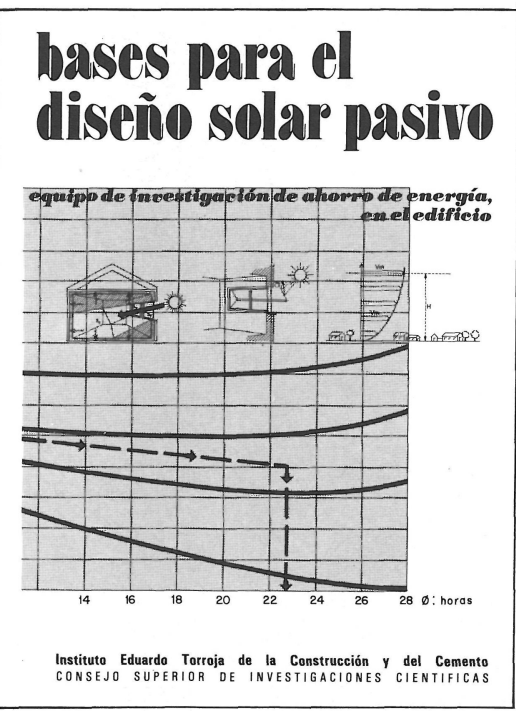

Equipo de Ahorro de Energía en el edificio

Dirección y coordinación: Arturo García Arroyo

M. ${ }^{a}$ José Escorihuela José Luis Esteban José Miguel Frutos Manuel Olaya Bernardo Torroja
Las dificultades de suministro y el alto coste de los productos energéticos convencionales han despertado la atención de los usuarios, técnicos e industriales de la edificación hacia los procedimientos y sistemas en que se basa el aprovechamiento de otras fuentes alternativas de energía, principalmente la solar. Esto ha generado un rápido desarrollo industrial $y$ comerial que, en opinión de los autores de de este libro, arrastran los sig un mimético tecnologismo respecto de los sistemas convencionales que violenta las peculiaridades de la energía solar (baja densidad y variabilidad en el tiempo), y una escasa selectividad en la aplicación de los sistemas y procedimientos pasivos dando origen a un ecumenismo arquitectónico solar, al margen de las condiciones climáticas y funcionales específicas de cada caso y lugar.

En este libro, utilizando criterios y metodología pedagógicos, se dan los fundamentos e instrumentos teórico-prácticos necesarios para el planteamiento de todo proyecto arquitectónico solar pasivo, de acuerdo con los principios éticos y económicos de conservación y ahorro de energía. Es decir: respeto de los presupuestos bioclimáticos, búsqueda de la máxima captación y acumulación de la radiación solar, y esmero en el aislamiento térmico de los cerramientos.

Un volumen encuadernado en cartulina ibiza plastificada, a cinco colores, de $16 \times 23 \mathrm{~cm}$, compuesto de 216 páginas, 217 figuras, 87 gráficos, 19 tablas y 10 cuadros.

Madrid, 1983. Precios: España 2.100 ptas.; 30 \$ USA.

\section{publicación del i.e.t.c.c.}

código-modelo ceb-fip para las estructuras de hormigón
El Instituto Eduardo Torroja, miembro activo tanto del Comité Eurointernacional del Hormigón (CEB), como de la Federación Internacional del Pretensado (FIP), ha tomado a su cargo la traducción y edición de esta importante normativa.

Aunque presentado con el título de "Código Modelo CEB/FIP 1978» este documento incorpora los dos primeros volúmenes de este "Sistema Unificado Internacional de Reglamentación Técnica de Ingeniería Civil». El primer volumen de este "Sistema Unificado» es el denominado "Reglas comunes Unificadas para los diferentes tipos de obras y materiales», donde se exponen los criterios y formatos de seguridad a que han de ajustarse los diferentes
Códigos (estructuras de hormigón, estructuras metálicas, estructuras mixtas, estructuras de albañilería y estructuras de madera), que han de configurar la totalidad del antedicho sistema.

El segundo volumen es propiamente el Código Modelo para las Estructuras de Hormigón. Fruto de la colaboración de dos asociaciones del prestigio del CEB y la FIP, desde mediados de los 60 , incorpora los avances científicos y tecnológicos producidos en los últimos años sin detrimento alguno de la claridad y operatividad que deben presidir un código que pretende ser, ante todo, un auxiliar práctico para los técnicos de la construcción.

El Código sigue en su estructura las reglas más o menos clásicas: una primera parte dedicada a los datos generales para el cálculo (propiedades de los materiales, datos relativos al pretensado, tolerancias); en segundo lugar se presentan las reglas de proyecto estructural (acciones, solicitaciones, estados limites últimos y de utilización, reglas de detalle para el armado); y, por último, ejecución, mantenimiento y control de calidad.

También incluye reglas para estructuras con elementos prefabricados y estructuras de hormigón con áridos ligeros. Los Anejos del Código se refieren a: terminologia, proyecto mediante la experimentación, resistencia al fuego, tecnología del hormigón, comportamiento en el tiempo del hormigón y fatiga.

Un volumen encuadernado en cartoné, de $21 \times 30 \mathrm{~cm}$, compuesto de 340 páginas, Madrid, mayo 1982. Precios: España 2.500 ptas. Extranjero 36 \$ USA. 